\title{
HIGH VOLTAGE CERAMIC CAPACITORS
}

\author{
D. A. NICKER \\ Erie Electronics Ltd., South Denes, Great Yarmouth, Norfolk, U.K.
}

(Received November 29, 1973; in final form May 18, 1974)

\begin{abstract}
The effects of voltage, temperature and time on ceramic dielectrics are summarized and general relationships between formulation, permittivity and stability are described. Factors affecting the electric strength of ceramics are outlined and suggestions made as to the breakdown mechanisms involved both within the ceramic and at the ceramic surface. The article is concluded with a description of commercial production techniques employed to overcome the potential hazards reported earlier.
\end{abstract}

\section{INTRODUCTION}

The ceramic capacitor employs a dielectric which is a mixture of inorganic, non-metallic materials, shaped in the powder state and sintered at temperatures of the order of $1,200^{\circ} \mathrm{C}$ to form an integral polycrystalline structure. Loosely, high voltage types may be considered to be those designed to operate at $1 \mathrm{KV}$ and above, but the dielectrics used differ little from those which satisfy applications at less elevated potentials.

Dielectric formulations available today offer permittivities from 15 to 15,000 but, unfortunately, each has a permittivity which will vary with factors such as temperature, voltage frequency and time, and generally stability decreases as the "initial" or "room temperature-zero volt" permittivity increases.

\section{CLASSIFICATION OF DIELECTRICS}

A variety of specifications for the classification of ceramic dielectrics exist, but all are similar in that it is the variation of "zero-volt" permittivity with temperature that is used to identify dielectrics. Two classes of dielectric, universally recognized, are:

Type 1. Dielectrics of high stability, especially suited for resonant circuit applications where low losses are important.

Type 2. Dielectrics for use in applications where stability and losses are not critical.

The materials of Type 1 are the temperature compensating dielectrics, each of which is identified by a temperature coefficient (TC), which is its variation of permittivity with temperature, from a value at $20^{\circ} \mathrm{C}$, expressed in parts per million per degree Centigrade $\left(\mathrm{ppm} /{ }^{\circ} \mathrm{C}\right)$. This variation may be considered essentially linear over the range $-55^{\circ} \mathrm{C}$ to $+125^{\circ} \mathrm{C}$ and, covered by the more common specifications, are temperature coefficients from $+100 \mathrm{ppm} /$ ${ }^{\circ} \mathrm{C}$ (P100) through zero (NPO) to $-1500 \mathrm{ppm} /{ }^{\circ} \mathrm{C}$ (N1500). Similar dielectrics are available with temperature coefficients of up to $-5600 \mathrm{ppm} /{ }^{\circ} \mathrm{C}$ and permittivities range from 15 for $\mathrm{P} 100$ to approximately 150 for N1500 and 800 for N5600. Dielectrics of this type may be considered to have permittivities which are not significantly affected by factors other than temperature.

Type 2 dielectrics are the high permittivity or $\mathrm{Hi}-\mathrm{K}$ ceramics, whose permittivities are usually over 250 and whose temperature characteristics are non-linear. Classification is made by the maximum allowed "zero-volt" permittivity excursion over a specific temperature range, expressed as a percentage of the permittivity at $20^{\circ} \mathrm{C}$. Manufacturers, however, will usually identify these materials by a formulation code which may incorporate an indication of the appropriate initial permittivity, e.g. K8000.7,8,10

\section{TEMPERATURE CHARACTERISTICS}

The high permittivity formulations employ barium titanate as the major constituent, the temperature characteristic of which is shown in Figure 1. Peak permittivity occurs at approximately $120^{\circ} \mathrm{C}$, which is known as the Curie point of the material but, at room temperature, a permittivity of approximately 1,500 is demonstrated. This level is closely associated with a high dissipation factor, which renders the material, in 




FIGURE 1 Barium titanate characteristics. The variation of permittivity $(K)$ and dissipation factor $(\tan \delta)$ with temperature.

its unadulterated form, unsuited to capacitor applications. Via substitutions of lead, strontium, calcium and cadmium for the barium ion, and similar substitutions of tin, zinc, or cerium for the titanium ion, the Curie temperature of the base material may be lowered and the degree of temperature dependence of permittivity reduced. A wide range of temperature characteristics is therefore rendered available, and typical examples are shown in Figure 2, which also serves to illustrate the point that stability is only available by a depression of permittivity.
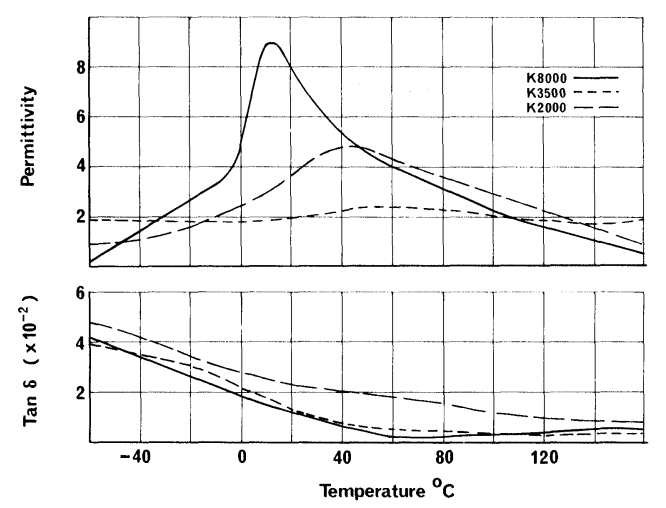

FIGURE 2 High permittivity ceramic dielectric characteristics. The variation of permittivity $(K)$ and dissipation factor $(\tan \delta)$ with temperature for three typical materials.

Additives, known as "shifters" or "depressors" according to their function, also have the effect of reducing losses, and dissipation factor is more a function of the particular additives used than of the initial permittivity. Insulation resistance, however, tends to fall as initial permittivity increases, and changes in temperature affect both parameters.

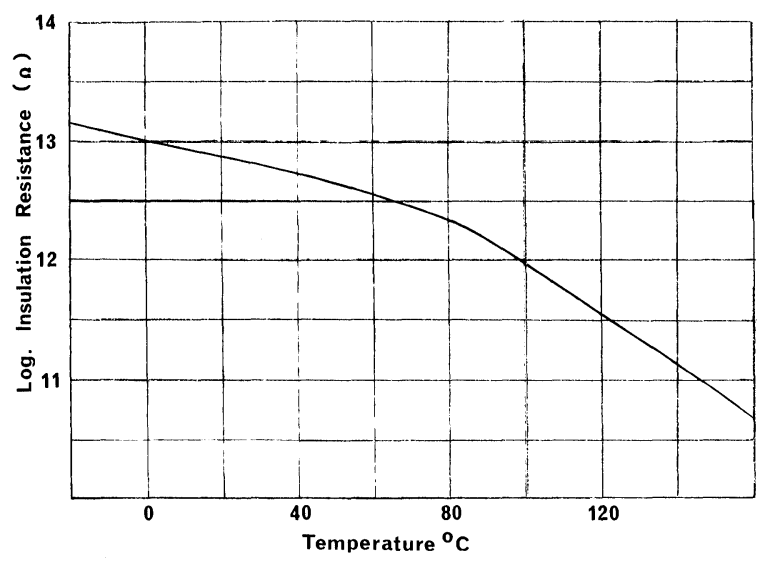

FIGURE 3 High permittivity ceramic dielectric characteristics. The variation of insulation resistance with temperature for a typical material.

Dissimilar, in one respect, to other dielectric systems, ceramic materials exhibit a decrease in dissipation factor with increasing temperature, an important factor with respect to a.c. working stresses. A semi-logarithmic decrease of insulation resistance at the higher temperatures is also usual, but the lowest value under normal working conditions is, in most cases, sufficiently high to be disregarded as a design factor (Figure 3).

\section{VOLTAGE EFFECTS}

Above its Curie point, the structure of barium titanate consists of a cubic array of barium ions, with an oxygen atom centrally disposed in each face. The titanium ion is situated at the intercept of the interstices of the octahedral array of oxygen ions, and the structure is non-polar. Below the Curie point, however, a transition occurs in which the structure becomes tetragonal and the titanium ion is displaced towards an oxygen ion, giving a dipole moment to the molecular group.

The electrostatic energy of the structure is minimised by the formation of domains, but the position of the titanium atom may be affected by an applied field, and domain growth occurs in the direction of the field. The changes in domain boundaries account for the variation in permittivity of titanate based materials with voltage, temperature and time, and there is evidence to suggest that stable dielectrics of high permittivity are possible by ensuring that the grain size of the polycrystalline structure is of the order of the domain size. This, however, is not the case for most commercial materials. 
Domain growth may be equated to a dipole movement and, as reorientation occurs more slowly than the rate of change of field, a hysteresis affect may be demonstrated by relating polarisation to the applied field. An analogy can therefore be drawn with the B-H loop of a ferromagnetic material and ceramics operating below their Curie point are known as "ferroelectrics". Above the Curie point, linear characteristics are encountered, and such materials are referred to as "paraelectric" dielectrics. The transition between ferroelectric and paraelectric properties in multi-phase ceramic systems is, however, ill-defined. ${ }^{3,9}$

The effect of a d.c. voltage on a ferroelectric ceramic is to bring about a reduction in permittivity, except at very low stresses where a slight positive change may be encountered. The magnitude of the decrease, per unit applied field, expressed as a percentage of the initial permittivity, generally increases as the initial permittivity increases. (Figure 4). Temperature also affects the degree of change with voltage, so that the simple addition of temperature characteristic data to a permittivity obtained from a room temperature voltage characteristic may be misleading.
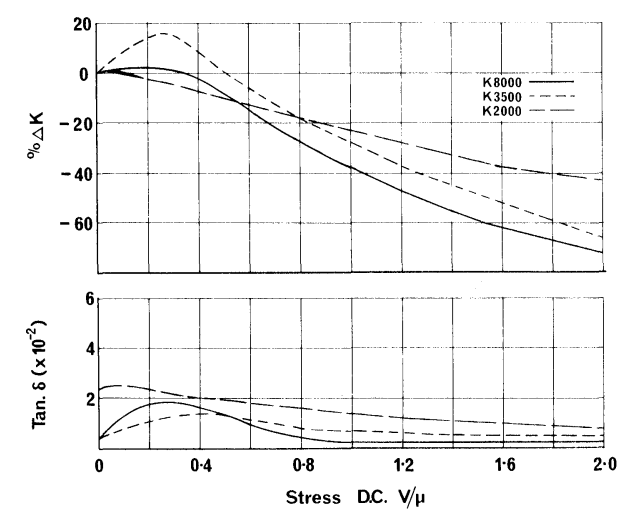

FIGURE 4 High permittivity ceramic dielectric characteristics. The variation of permittivity $(K)$ and dissipation factor $(\tan \delta)$ with applied d.c. stress, for three typical materials.

Whilst insulation resistance remains substantially unaffected, a reduction in permittivity with d.c. voltage is accompanied by a reduction in dissipation factor.

The effect of a.c. voltages on ferroelectric materials is somewhat more complex. Additives, employed to de-sensitise formulations to temperature and d.c. voltage, achieve this property by limiting grain growth, thereby inhibiting dipole movement, and high dissipation factors accompanied by lower permittivities maybe evident at higher frequencies. (Figure 5). Frequency sensitivity is therefore independent of the initial permittivity of dielectrics, usually considered to be that at $1 \mathrm{kHz}$.

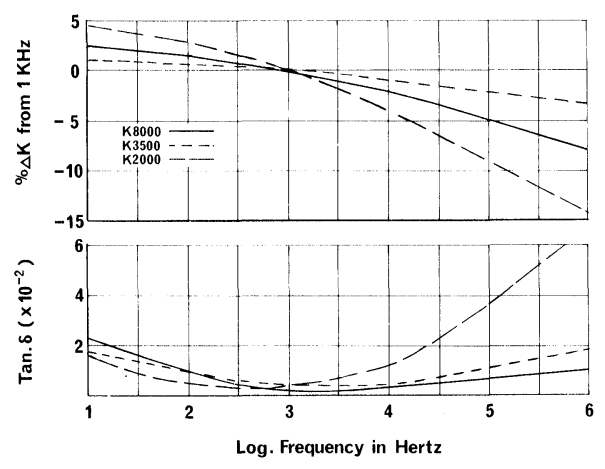

FIGURE 5 High permittivity ceramic dielectric characteristics. The variation of permittivity $(K)$ and dissipation factor $(\tan \delta)$ with applied frequency, for three typical materials.

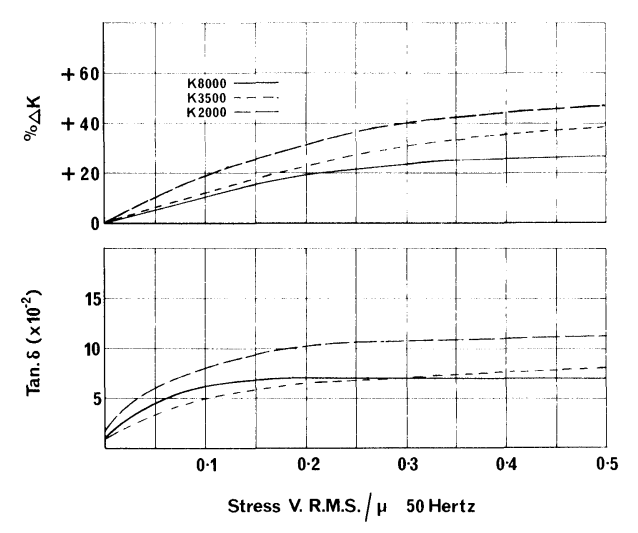

FIGURE 6 High permittivity ceramic dielectric characteristics. The variation of permittivity $(K)$ and dissipation factor $(\tan \delta)$ with applied a.c. stress for three typical materials.

A positive change in both permittivity and dissipation factor may be expected with increasing a.c. field strength, this again more a function of additives than of initial permittivity (Figure 6). Losses occurring may generate a significant temperature rise in the test piece, yielding a shift in both parameters via their temperature characteristics, until an equilibrium temperature is reached. Should this temperature exceed the dielectric Curie temperature, a decrease in permittivity may follow the initial increase. As the equilibrium temperature is not only a function of applied voltage and dielectric characteristics, but also 
a function of dielectric construction, insulation and environment, a.c. characteristics of finished devices are somewhat difficult to predict.

If capacitors are required to operate under stringent a.c. conditions, it is usual to employ a formulation whose Curie point has been shifted well below room temperature. Such materials, which operate well into the paraelectric region of the temperature characteristic, include the type 1 dielectrics. ${ }^{4,8}$

\section{AGEING}

Gradual domain reorientation in ferroelectric ceramics, after heat cycling, accounts for a minor loss of permittivity with time. Known as "ageing", the phenomenon is predictable and is quantified by expressing the loss as a percentage of the initial permittivity per logarithmic decade of time. The initial permittivity is considered to be that of the dielectric after one hour's recovery from $150^{\circ} \mathrm{C}$. Ageing rates increase with initial permittivity (Figure 7) and, where applicable, manufacturers, when quoting capacitances and tolerances of finished parts, usually base these on permittivity values at 1,000 hours beyond which further losses become insignificant in relation to other stability factors. Lost permittivity may be recovered by recycling capacitors through their Curie points, at which time ageing recommences.

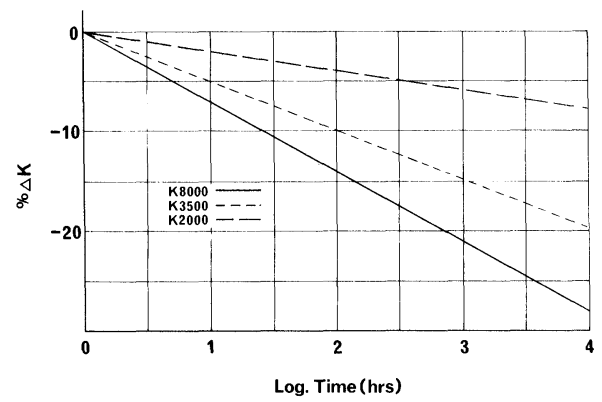

FIGURE 7 High permittivity ceramic dielectric characteristics. The variation of permittivity $(K)$ with time for three typical materials (ageing).

Accompanying a decrease in permittivity with ageing is a decrease in dissipation factor. An additional ageing factor, however, may be introduced by the effect of continuously applied d.c. voltages. Even if devices are operated above their Curie points, a decrease in permittivity over and above that which might be attributed to time-based ageing, may be noticed. (Figure 8). Positive changes are, however, not uncommon should loading be carried out at very high temperatures. Maximum permittivity excursions with applied voltage. temperature and time are outlined in manufacturers' specifications for individual formulations. ${ }^{4,9}$

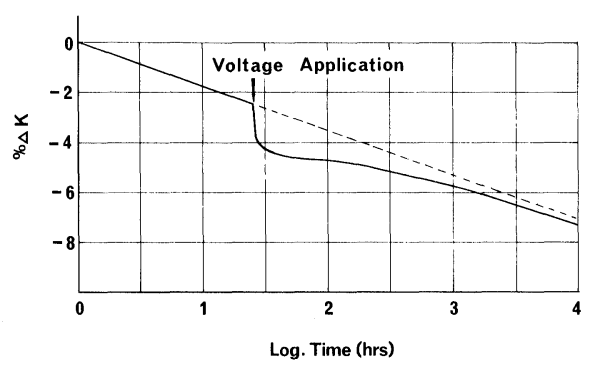

FIGURE 8 High permittivity ceramic dielectric characteristics. The affect of voltage application on the variation of permittivity $(K)$ with time for a typical material (voltage ageing).

\section{DIELECTRIC BREAKDOWN}

Continuous voltage ratings are arrived at on the basis of the results of load tests at stresses, frequencies and temperatures over and above those envisaged in the end-use application. The timing of failures under such circumstances enables a statistical prediction of expected life under normal conditions. Initial test levels are, however, derived from a working knowledge of a particular formulation's electric strength. This factor, which is by no means constant, is found to vary empirically with ceramic porosity, permittivity, temperature, thickness, electrode area, electrode shape and rate and duration of applied voltage.

The density of ceramic parts is inevitably less than that predicted from a consideration of raw material constituents, the difference being attributable to the occurrence of cavities throughout the polycrystalline structure of the fired pieces. Electric strength decreases as both the incidence and size of these "pores" increase and, typically, the electric strength of a dielectric with $14 \%$ porosity may be half that of a similar sample with 5\% porosity. Electric strength is therefore loosely related to fired density (Figure 9a).

Electric strength, in general, decreases as initial permittivity increases (Figure 9b), but a more exact relation might be achieved should permittivity at the applied stress and temperature be considered. 


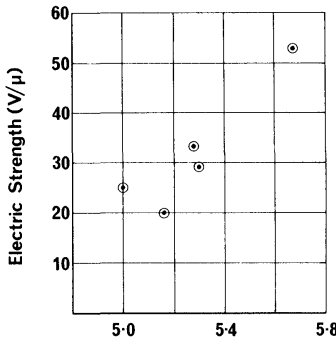

a) Density

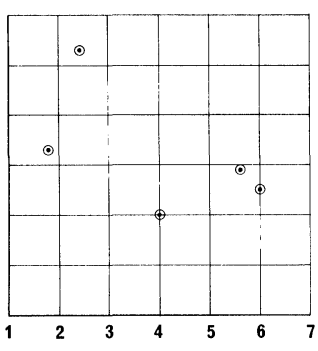

b) Initial Permittivity $\times 10^{3}$
FIGURE 9 High permittivity ceramic dielectric characteristics. The relationship between electric strength and (a) density and (b) initial permittivity for typical materials.

A relationship exists between electric strength and specimen thickness $\left(E \simeq k t^{-1 / 2}\right)$ as indicated by Figure 10. However, of more practical interest is the re-plot of these values as breakdown voltage against thickness. Clearly an increase in thickness will not yield a proportional increase in breakdown voltage.
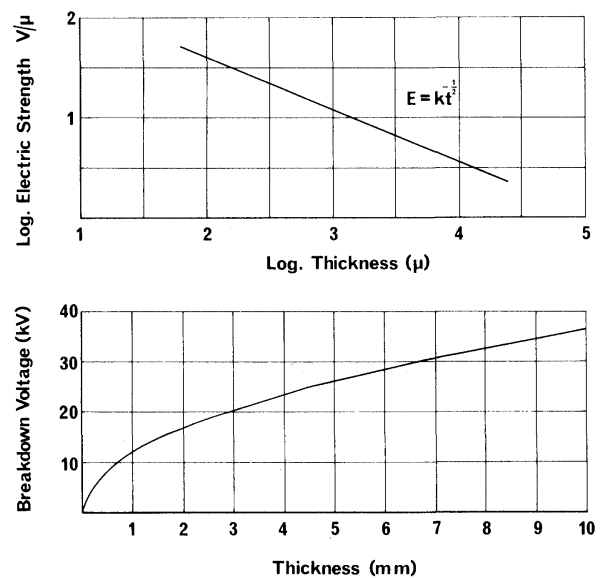

FIGURE 10 High permittivity ceramic dielectric characteristics. The affect of specimen thickness on (a) electric strength and (b) breakdown voltage for a typical material.

The voltages shown in Figure 10 are in excess of those that would be achieved on capacitors bulk manufactured from the same formulation, and the difference is largely attributable to voltage distribution within the dielectric. The use of electrodes of hemispherical configuration, which ensure a known maximum voltage stress may yield a threefold increase in apparent electric strength over commercial parts employing planar electrodes, where an illdefined equipotential concentration at electrode edges may occur (Figure 11). An increase in the area

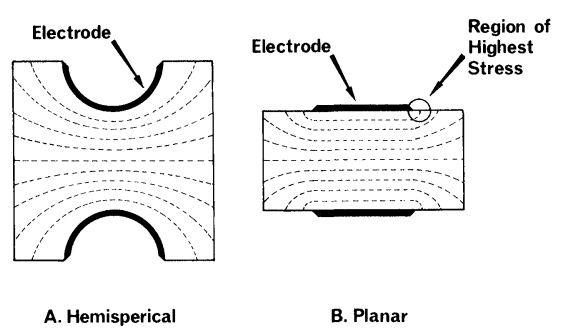

FIGURE 11 The voltage distribution within typical examples of ceramic dielectric configurations.

of planar electrodes may also bring about a minor decrease in electric strength if parts exceed a certain thickness.

The rate of application of voltage significantly affects the breakdown voltage of a ceramic part. Typically, d.c. electric strengths for rates of the order of $\mathrm{kV} / \mu \mathrm{sec}$ may be as low as one third of that for rates of the order of $\mathrm{kV} / \mathrm{min}$. Similarly, a.c. breakdown voltages may be of the order of half the d.c. breakdown voltage for the same rate of application and a further decrease may be expected with increasing frequency.

It can by no means be stated that the breakdown mechanism of ceramic materials is fully understood, but attempts have been made to explain variations in electric strength by attributing the onset of breakdown to discharges within the ceramic pores. A voltage applied to a dielectric produces a calculable stress across a cavity that is in excess of the stress across the dielectric itself. The cavity is air filled, and a discharge, internal to the cavity and between its walls, occurs when the stress reaches the breakdown stress of the equivalent air gap. It has been suggested that such a discharge results in considerable local heating in the immediate area of the discharge, initiating thermal breakdown of the crystal lattice, and a correlation between discharge inception voltage and breakdown voltage has been reported..$^{5,6}$

Owing to the complexity of obtaining absolute measurements with respect to the multitude of dielectric formulations available, manufacturers of commercial parts merely quote minimum breakdown voltages of finished components under specific conditions. These are usually well below the mean levels actually achieved and working voltages are seldom greater than $40 \%$ of this mean level. The resultant d.c. working stresses can vary from $3 \mathrm{~V} / \mu$ for some high density, low permittivity dielectrics, down to $1 \mathrm{~V} / \mu$ for higher permittivity, porous materials and, in many 
instances, the lower working stresses of the latter will preclude any apparent size advantages of these dielectrics. D.c. impulse and a.c. working stresses are reduced proportionately but, for general purpose applications, it is found that maximum permittivity, and therefore maximum volumetric efficiency, within a given voltage/temperature envelope, is usually satisfied by dielectrics with initial permittivities in the region 2000 to $5000.5,6,11$

\section{SURFACE EFFECTS}

Dielectric ratings are not the only factor in governing component size and often a minimum size limitation is placed on a component which is attributable to insufficiencies on the part of insulation systems available.

As stated previously, with dielectric configurations employing planar electrodes, high stresses exist in the dielectric at the electrode edge. A similar stress component, parallel to the dielectric surface, is present in any adjacent insulating medium. If the latter is air, discharges occur between the electrode edge and the dielectric surface, as the breakdown stress of the air is exceeded. The inception voltage of such discharges is predictable and, when measured between capacitor electrodes, is proportional to the square root of the dielectric thickness and inversely proportional to the square roots of both the permittivity and the ambient temperature. A minimum inception voltage of approximately $250 \mathrm{~V}$ rms is predicted for devices whose ratio of dielectric thickness $(\mathrm{mm})$ to permittivity is low $(<0.01)$. The length of a discharge under such conditions will be of the order of $10 \mu$, but an elevation of voltage will allow discharges to occur over both larger and smaller distances, following a gap/stress relationship similar to that predicted for air by the well known Paschen curve (Figure 12). At a given voltage, the device will short circuit across the dielectric surface. It is therefore essential to provide the dielectric with a high electric strength insulative coating. ${ }^{1,11}$

Insulative media used are organic resins, usually epoxide, phenolic or polyurethane based, whose properties have been tailored by the addition of inorganic materials, to meet any additional environmental or cosmetic requirements envisaged for the application.

A problem is presented, however, by the ceramic/ insulation interface, as in this region cavities may be introduced by limitations on the part of the manufacturing process, or materials used, or both. Such

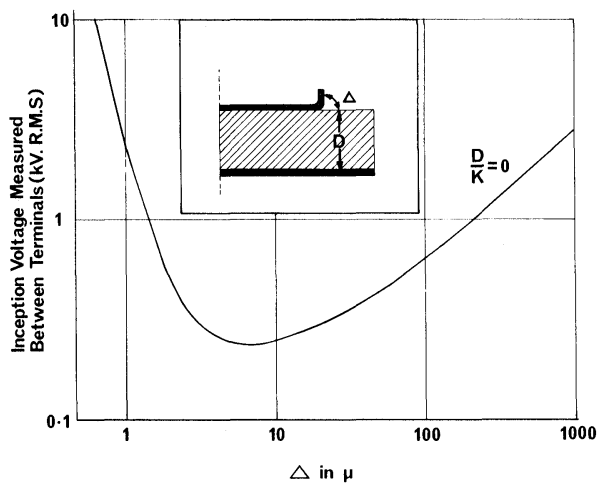

FIGURE 12 Inception voltage as a function of the length of the gap discharged at the surface of a high permittivity dielectric.

regions will be subject to internal discharges, but it is noteworthy that ionization in this region does not affect the breakdown voltage of the ceramic dielectric itself. The capacitor may therefore be operated above the inception voltage of "surface discharges" if insulation is selected that is capable of withstanding the mechanical, thermal, chemical and radiative effects of the ionic bombardment involved.

An injudicious choice of insulation will facilitate the erosion of a cavity into a pit radiating from the electrode. As the discharges continue, the material carbonises and the pit is rendered conductive. It therefore acts as an extension of the electrode, generating very high stresses at its tip. Should the stresses at the tip exceed the intrinsic electric strength of the material, breakdown occurs over a short distance. The pit is therefore extended, the field concentration moves to the new tip and a narrow channel propagates rapidly along the interface which may eventually succeed in short circuiting the capacitor. This failure mode is sometimes referred to as "tracking". The rate of deterioration increases with the number of discharges and is consequently proportional to the frequency of the applied voltage and its magnitude. It also increases with the magnitude of the discharges, which is proportional to the shape and size of the cavities.

The initial erosion of the cavity forms the major part of the time to surface breakdown, which may be anything from a few minutes to a great many years. Judiciously chosen capacitor insulation will withstand a high level of continuous internal (surface) discharges, although of course the envisaged application will dictate the maximum tolerable level. It is unusual for high voltage capacitors to be totally discharge free at their working voltage but, should 
such a requirement arise, freedom from discharges can be assured by a reduction in stress level via an increase in size.

\section{PRODUCTION TECHNIQUES}

Relative sizes of typical capacitors for use at different d.c. working voltages are demonstrated in Figure 13. All dielectric slugs used in the samples shown are of disc form although, at lower voltages, this configuration may be supplemented by rectangular or tubular types. ${ }^{7,8}$

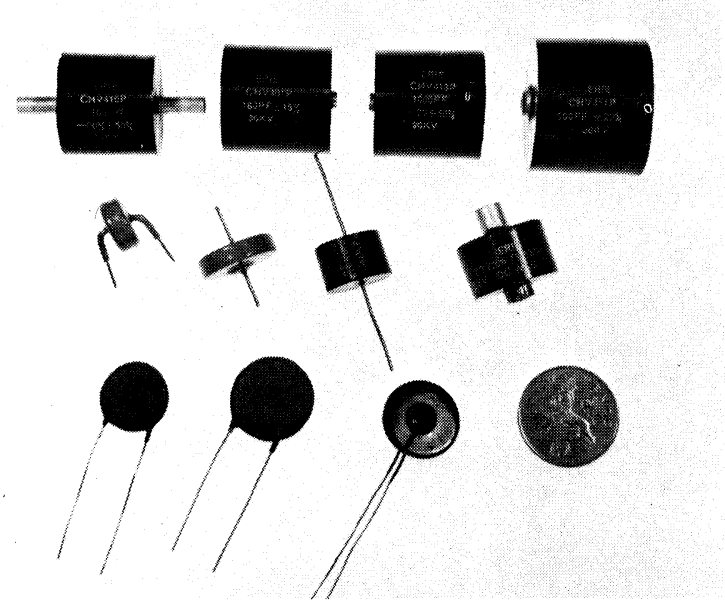

FIGURE 13 Typical examples of commercially available high voltage ceramic capacitors.

Electrodes are usually silver powder or flake dispersed in a glass medium. Applied in the form of a paste after the ceramic firing cycle, the material is fired onto the ceramic at temperatures just below the melting point of the metal content. A dispersion of discrete metal particles is therefore held onto the dielectric by the fusion of the glass "frit", thus assuring an intimate electrical contact with the ceramic, whilst presenting a readily solderable surface for the application of metal terminations.

At the lower voltages, planar electrodes are applied to the discs by silk screening. Wire leads are affixed radially, by immersing the disc, held between the formed leads, in molten solder. Subsequently cured insulation is applied by dipping in liquid resins, usually heavily filled phenolics, which may be waximpregnated at a later stage to assist protection against humidity. Alternatively, conformal insulation may be applied by immersing the preheated disc in epoxide resin powder fluidised by air injection. The partially adhering powder coating is then reflowed and cured by further heat treatment.

At higher voltages, the difference in potential between the capacitor leads and the dielectric surface, peripheral to electrode, may allow an excessively high level of discharge in the intervening insulation. It is therefore common to introduce a slight kink in the radial lead, elevating it from the dielectric surface so as to reduce stresses in this region.

At voltages of the order of $8 \mathrm{kV}$, it is vital that the lead be kept clear of the ceramic surface and axial leads are employed. To retain the advantage of high component density on the circuit board, the leads may be reformed to a radial configuration.

Wire ended components are seldom supplied for use in air at voltages above $10 \mathrm{kV}$ d.c. as, at this level, corona discharge may occur in the highly stressed region about the leads. Circuit manufacturers, however, retain the economic benefits gained by the use of wire ended components, by encapsulating assemblies in epoxide or polyurethane resin systems, or in silicone rubbers. Capacitor finishes are therefore varied to suit and light finishes, applied by liquid or electrostatic sprays, are available which do not add significantly to the dimensions of the disc. Continuous unprotected use is precluded by virtue of the minimal thickness of the insulation. However, ease of handling is assured and a facility of limited circuit test, prior to encapsulation, offered. This type of finish also relieves the encapsulant of the necessity of total protection against tracking.

Above $10 \mathrm{kV}$, dielectric dimensions are such that conformal coatings become difficult to apply, and encapsulants are employed should individual capacitors be required for unprotected use in air. Terminations with a large radius of curvature are essential and turned brass parts, silver plated and provided with tapped inserts to enable screw mounting of the heavy finished parts are used.

Alternative electrode configurations may be employed to afford a measure of stress relief to the dielectric. One principle involves electroding the total area of the planar surfaces of the disc, thus ensuring that the ares of highest stress are removed from the ceramic into the higher electric strength region of the insulation. An alternative system partially duplicates the hemispherical electrode of laboratory test pieces, a well being achieved either by turning the dielectric in the unfired state, or by impressing it during the forming operation of the slug. Metallising for both systems is applied by hand painting or spraying. 
It is unusual to obtain ceramic capacitors, for use in air at voltages in excess of $40 \mathrm{kV}$ d.c., that employ a single ceramic slug. Dielectric thickness required are of the order of $30 \mathrm{~mm}$, necessitating diameters of up to $60 \mathrm{~mm}$ and such pieces may well weigh as much as half a kilogram. High density formulations shrink up to $20 \%$ on firing and, at this order of size, inordinate precautions are necessary in order to prevent distortion and maintain uniform density. Above $40 \mathrm{kV}$, a capacitor requirement is usually fulfilled by employing an assembly of discrete, lower rated components in series, or series parallel connections.

\section{REFERENCES}

1. F. H. Kreuger, Discharge Detection in High Voltage Equipment (Heywood, London, 1974).

2. J. C. Anderson, Dielectrics (Chapman and Hall, London, 1964).
3. W. D. Kingery, Introduction to Ceramics, (John Wiley, New York, 1960).

4. J. H. Burton and E. A. Bolton, "A better understanding of ceramic capacitors", Electron Compon. 10, 941 (1969).

5. G. J. Hill, The Electric Strength of High Permittivity Ceramics, E.R.A. Report No. 70-68 (1970).

6. G. J. Hill, Energy Storage in Ferroelectric Ceramics Survey of Materials Based upon Barium Titanate, E.R.A. Report No. 69-35 (1969).

7 J. B. Birks, Modern Dielectric Materials (Heywood, London, 1960).

8. G. W. A. Dummer, Fixed Capacitors (Pitman, London 2nd ed. 1964).

9. J. M. Herbert. "Survey: Ferroelectric ceramic materials." Compon. Technol. 4, 17 (1971).

10. BS 9070: Section 5: 1971. Specification for Fixed Ceramic Capacitors of Assessed Quality: Generic Data and Methods of Test. Section 5. Ceramic Dielectric Capacitors. (British Standards Institute, 1971).

11. S. Whitehead, Dielectric Breakdown of Solids. (Clarendon Press, Oxford, 1951). 

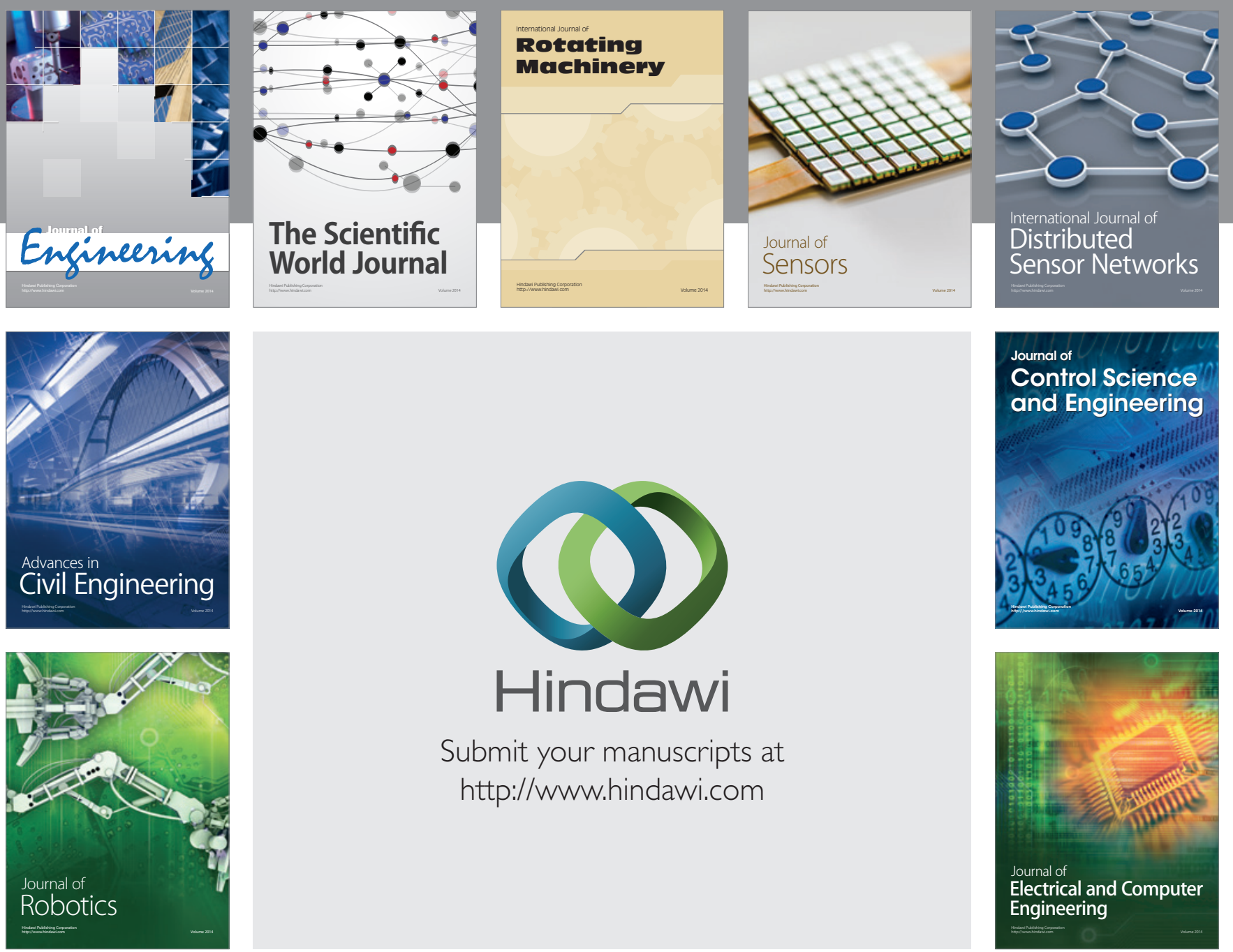

Submit your manuscripts at

http://www.hindawi.com
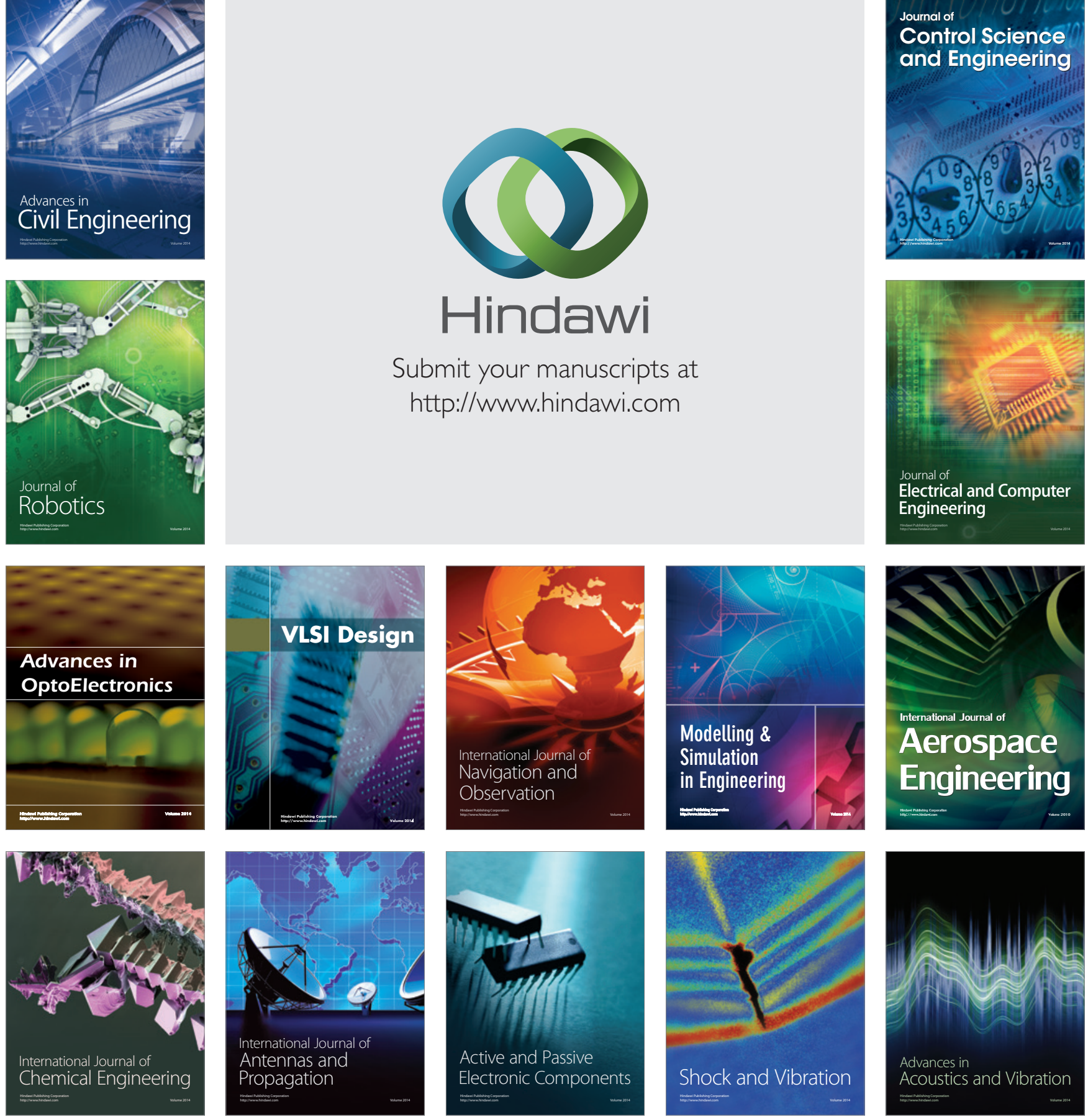